\title{
e-Phaïstos
}

e-Phaïstos

Revue d'histoire des techniques / Journal of the history

of technology

VII-2 | 2019

La sauvegarde du patrimoine scientifique

\section{Agglomérés, agglomérer : Valoriser les charbons non marchands, en Europe, au XIX ${ }^{\mathrm{e}}$ siècle}

Agglomerate: Giving Value to Non-merchantable Coals in Nineteenth-century

Europe

Jean-Philippe PASSAQUI

\section{CpenEdition}

Journals

Édition électronique

URL : http://journals.openedition.org/ephaistos/5436

DOI : $10.4000 /$ ephaistos.5436

ISSN : 2552-0741

Éditeur

IHMC - Institut d'histoire moderne et contemporaine (UMR 8066)

Référence électronique

Jean-Philippe PASSAQUI, « Agglomérés, agglomérer : Valoriser les charbons non marchands, en Europe, au XIXe siècle», e-Phaïstos [En ligne], VII-2 | 2019, mis en ligne le 03 octobre 2019, consulté le 06 novembre 2019. URL : http://journals.openedition.org/ephaistos/5436 ; DOI : 10.4000/ephaistos. 5436

Ce document a été généré automatiquement le 6 novembre 2019

Tous droits réservés 


\title{
Agglomérés, agglomérer : Valoriser les charbons non marchands, en Europe, au XIX ${ }^{\mathrm{e}}$ siècle
}

\author{
Agglomerate: Giving Value to Non-merchantable Coals in Nineteenth-century \\ Europe
}

Jean-Philippe PASSAQUI

1 Valoriser les déchets, les rendre marchands, c'est-à-dire commercialisables, l'enjeu a toujours prévalu depuis l'avènement du capitalisme industriel, surtout quand ces mêmes déchets correspondent à une fraction importante de la production et qu'ils peuvent avoir des conséquences néfastes sur l'organisation productive. Au cours du XIX ${ }^{e}$ siècle, la grande industrie a souvent été confrontée à l'existence de ces déchets, conséquence de sa propre activité. C'est pourquoi, dans la sidérurgie par exemple, l'utilisation des chutes de métal a été précoce et intensive, au point d'ailleurs d'occuper une place prépondérante dans l'avènement de certains procédés sidérurgiques. On pense à l'acier Martin notamment.

2 Pour les compagnies houillères, le problème de la gestion des déchets de l'extraction est encore plus évident, car ils ne représentent pas qu'un coût. Ils sont aussi source de perturbation de l'exploitation au point de compromettre l'existence d'une mine de charbon. Ferdinand Chalmeton, directeur des houillères de Bessèges, résume ainsi le défi industriel commun aux entreprises minières du Gard, de l'Hérault, mais aussi du Centre de la France :

\footnotetext{
«Généralement, les couches de houille sont faibles et éloignées les unes des autres, ce qui nécessite des travaux longs et coûteux. L'impureté du charbon occasionne la création de moyens mécaniques d'épuration dispendieux ${ }^{3}$.»
}

3 Il évoque le traitement mécanique de la houille, mais le problème couvre d'autres stades de sa valorisation. Comme souvent, la technique de l'agglomération de la houille 
apparaît, de manière plus ou moins spontanée, dans plusieurs bassins industriels d'Europe. Si Britanniques, Belges et Allemands s'intéressent à la question, c'est d'abord en France que les efforts les plus constants et les plus probants sont menés. Ils sont le fait des principales exploitations du Centre et du Midi de la France : la Grand-Combe, dans le Gard, les différentes mines de Saint-Étienne, dans la Loire, et les houillères de Blanzy, en Saône-et-Loire ${ }^{4}$. Dans le Nord, quelques compagnies font de ce type de fabrications une spécialité, Anzin et à Aniche notamment.

Figure 1. Briquettes de houille produites par différentes compagnies minières.

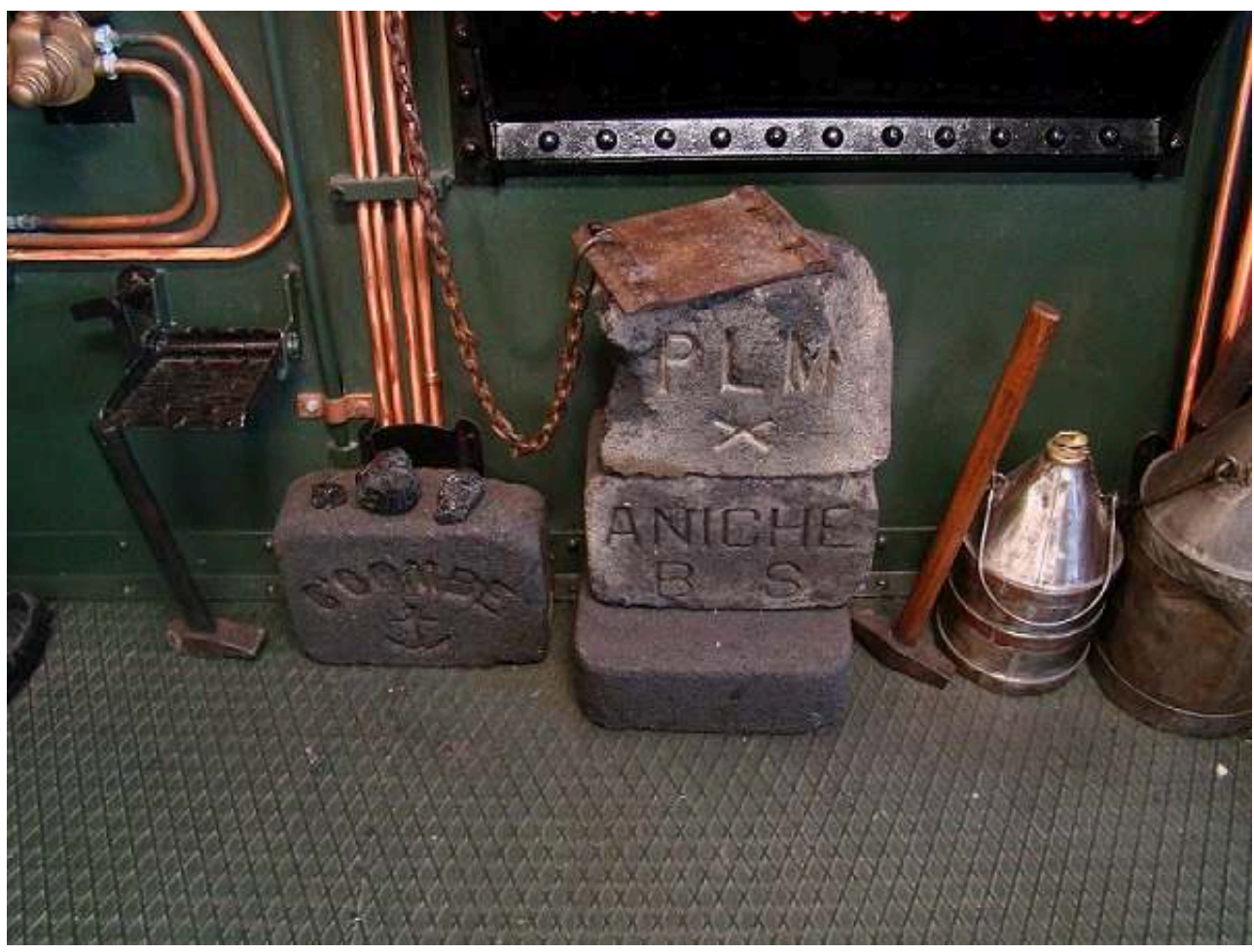

Les angles sont émoussés au moment du moulage pour éviter le bris et la perte de combustible. Comme les briques sont obtenues par moulage, il est possible, pour les compagnies productrices de faire figurer le nom et parfois le logo de la compagnie productrice. L'ancre présente sur la briquettes de la Grand' Combe rappelle que les premiers usages ont été destinés à la navigation.

(c) Jean-Philippe Passaqui

C'est aussi un moyen pour assurer une reconversion et une réorientation industrielles précoces après la perte d'un important client, comme dans le cas des mines de Brassac, en Auvergne. Elles se lancent dans l'agglomération de la houille au profit des compagnies de chemins de fer au moment même où elles perdent, avec les usines de la Nièvre, leurs principaux débouchés pour leur coke métallurgique ${ }^{5}$.Mais pourquoi l'effort a-t-il été aussi soutenu dans le Centre de la France au point d'en faire le district industriel de référence pour cette activité?

\section{Dépasser le manque de régularité des combustibles minéraux}

Plusieurs aspects entrent en considération. Ils rendent cette localisation logique. Les charbons y sont souvent beaucoup plus irréguliers que dans les autres bassins houillers 
européens, au niveau de leur teneur en cendres et de leur pouvoir calorifique. Or, au milieu du XIX ${ }^{e}$ siècle, à la clientèle domestique, à celles des petites industries qui se satisfont de charbons à la composition irrégulière, s'ajoutent celles des compagnies de chemins de fer, de navigations fluviale et maritime, et des entreprises sidérurgiques comme de constructions mécaniques. Elles ont besoin de connaître précisément les caractéristiques des combustibles solides qu'elles consomment. Pour ce faire, elles exigent la rédaction de contrats d'une grande précision auxquels les compagnies minières peinent à répondre de manière satisfaisante. C'est qu'un combustible moins cendreux permet de produire, à masse équivalente, plus de vapeur et donc d'aller plus vite et plus loin.

Or, dans le même temps, les exploitations du Centre et du Midi sont confrontées à la problématique des charbons menus. Fragments de petits calibres, ils ont toujours représenté une part importante de l'extraction, mais cette proportion est croissante dès lors que les mineurs cheminent dans des quartiers déjà exploités où les couches sont broyées. C'est aussi une conséquence de la modification des méthodes d'exploitation qui, à partir des années 1840-1850, a tendance à disloquer les charbons des dernières tranches de chaque étage. Surtout, depuis les débuts de l'extraction de la houille, ces charbons menus, considérés comme invendables ou à des prix inférieurs à leur coût d'extraction, sont abandonnés dans les chantiers. Les tarifs de ces combustibles restent à des niveaux très bas tant que la clientèle est incapable d'utiliser d'autres charbons que ceux de gros calibres. La faible rentabilité des houillères, la médiocrité de l'environnement commercial dans lequel elles se débattent, l'élévation régulière des besoins d'investissement à mesure que les travaux s'approfondissent concourent à repousser toute initiative en vue de favoriser l'emploi des charbons à faible granulométrie.

7 En prenant l'habitude de les abandonner au fond, les industriels ont pris le risque que ces poussières et petits fragments de charbon, qu'ils considèrent comme des déchets, s'échauffent par réactions chimiques ou biologiques internes, en produisant de l'oxyde de carbone dangereux pour les mineurs. Ils peuvent même entrer en combustion, spontanément, au contact de l'oxygène contenu dans l'air. C'est ce qui se produit régulièrement dans des mines comme Commentry, Le Creusot, Longpendu, Decazeville et, bien sûr, dans les différentes exploitations de Saint-Étienne ${ }^{6}$. En parallèle, nous l'avons constaté, une nouvelle clientèle apparaît, importante par les volumes commandés, exigeantes par les qualités demandées. Dès lors qu'il devient indispensable de retirer tous les charbons du fond, pour éviter le retour des incendies qui désorganisent voire interrompent la production, l'idée de trouver un usage à des combustibles auparavant délaissés est tentant. En outre, leur volume augmente pour des raisons corollaires de celles déjà évoquées. Avec cette clientèle industrielle indispensable, mais qui inflige de lourdes pénalités quand les teneurs en cendres excèdent celles spécifiées dans les contrats, la préparation de la houille doit devenir une priorité. Elle représente un coût que la mécanisation peut atténuer. La valorisation se décompose en deux étapes. Le triage s'opère surtout sur le carreau même des puits, pour éliminer les matières non combustibles. Il est accompagné du lavage, avec la création, dans les années 1850-1860, des premières grandes installations mécanisées ${ }^{7}$. Il en découle une production supplémentaire de poussières charbonneuses et de petits fragments de houille. Mal adaptés aux chaudières existantes, ils constituent pourtant, 
du point de vue du pouvoir calorifique et de la teneur en cendres, de remarquables combustibles... potentiels ${ }^{8}$.

D'où l'idée d'agglomérer ces charbons, en prenant appui sur l'exemple de la tourbe, autre combustible. Mais la seule compression ne peut suffire pour agréger les particules de houille. Les expériences menées par Maximilien Évrard, à partir de son premier dispositif d'agglomération, ont démontré que la production de briquettes sans liant n'est envisageable que dans les cas où elles sont consommées sur place, ce qui est en opposition avec les caractéristiques de la clientèle, composée essentiellement de compagnies de transport. Avec l'association entre la houille et un liant, que Louis Grüner nomme un ciment, destiné à donner davantage de cohésion au produit ${ }^{9}$, il s'agit tout bonnement de mettre au point une technique capable de fabriquer des « charbons artificiels ». L'emploi d'un liant est décrié par les consommateurs, en raison des fumées qu'il dégage, et de la gêne que cela provoque auprès des chauffeurs comme de la clientèle.

9 C'est le combustible stéphanois qui s'impose comme le plus apte à l'agglomération. Au début des années 1860, les comparaisons effectuées à ce sujet par la Marine impériale, en attestent ${ }^{10}$. Mais cela ne suffit pas à expliquer pourquoi le bassin et le charbon stéphanois ont porté les premiers pas de cette industrie en France, et, au-delà, en Europe.

\section{Saint-Étienne, un district industriel innovant}

10 Saint-Étienne n'est pas qu'un bassin houiller. D'autres activités, la sidérurgie et les constructions mécaniques, s'y sont implantées et sont en plein développement au cours des années 1850-1860. Le vivier d'ingénieurs est étoffé et apte à innover. Parmi les partisans de l'agglomération de la houille en France figurent notamment deux anciens directeurs de l'École des mines de Saint-Étienne, Charles Combes ${ }^{11}$ puis LouisEmmanuel Grüner. Le premier incite les industriels à se doter des équipements nécessaires, en garantissant que les débouchés ne manqueront pas. Son intervention auprès des mines de la Grand-Combe est d'ailleurs décisive. Le second fait un tour d'horizon des différentes méthodes, afin que son évaluation incite les exploitants français à se tourner vers une technique d'agglomération qui soit la moins gourmande possible en brai. Car il convient de séparer deux aspects, la technique d'agglomération et l'outillage pour agglomérer. Souvent, ils se sont retrouvés réunis par des ingénieursdirecteurs ayant conçu en même temps les dispositifs d'agglomération et les équipements ad hoc. Dans ce domaine, les anciens élèves de l'École des mines de SaintÉtienne s'imposent par leur compétence et leur capacité à imaginer non seulement les presses à agglomérer, mais aussi l'ensemble des équipements associés. Trois noms se détachent: Evrard, Révollier et dans une moindre mesure Bérard, tous passés par les bancs de l'École des mines de Saint-Étienne. Au milieu du XIXe siècle, ils ont façonné l'industrie de l'agglomération et, plus généralement, toute la chaîne accompagnant la mise en valeur de la houille.

11 Révollier n'a pas laissé une trace visible dans l'histoire de la mise au point des premiers équipements de machines à agglomérer. Marsais, un autre ancien élève de l'École des mines de Saint-Étienne, lui a été préféré ${ }^{12}$. Ils partagent pourtant la paternité de la première installation érigée dans un but industriel et le fait d'avoir donné l'impulsion à cette nouvelle activité. Le parcours de Marsais ne le préparait pas à construire 
l'outillage nécessaire, mais il faisait de lui un remarquable observateur des besoins de l'industrie. Breveté de l'École des mineurs de Saint-Étienne, sa carrière d'ingénieur n'est pas sans mérite. Il s'est signalé par la mise au point de plusieurs équipements de transport dans les mines, préfiguration de l'importance donnée à la circulation autonome des matières dans les ateliers d'agglomération. C'est comme directeur d'une houillère du bassin stéphanois qu'il en est venu à s'intéresser à l'utilisation des menus, au point d'être récompensé, en 1849, par une médaille d'argent à l'exposition de Paris ${ }^{13}$. Pourquoi l'avoir associé à J-F. Révollier ? La première machine à agglomérer utilisée à Saint-Étienne est en fait le fruit d'un partenariat avec l'entreprise de ce dernier. Par la suite, la démarche de Révollier n'est pas très éloignée de celle d'Evrard. Mais s'il ne dirige pas une mine, il est à la tête d'un des plus grands ateliers de constructions mécaniques de France. Révollier ne se contente pas d'imaginer les équipements d'agglomération. Il y associe des installations de lavage de la houille, pour lesquelles il prend un brevet en $1861^{14}$. Un de ses principaux ingénieurs, chef de bureau des études, Couffinhal, est connu pour avoir mis au point une presse qui devait prendre son nom ${ }^{15}$.

13 Grâce à la qualité des produits obtenus, la machine mise au point par Marsais et Révollier devait être utilisée sans grand changement jusque dans les années 1870 et essaimer dans plusieurs pays, notamment en Belgique et dans les États allemands. Mais, comme souvent avec les équipements industriels conçus dans les années 1830-1840, la machine à agglomérer de Révollier est encombrante et d'un fonctionnement complexe. Dès lors que des équipements concurrents sont éprouvés et proposés aux compagnies charbonnières, elle apparaît dépassée. L'entreprise fondée par Révollier ne s'en désintéresse pas pour autant. Entretemps, elle est devenue un partenaire indispensable des houillères soucieuses d'améliorer la qualité de leurs produits.

Rompant avec sa logique antérieure, qui consistait à se contenter d'améliorations de détails sur un équipement éprouvé, elle s'engage dans la conception d'un équipement totalement repensé et redevient compétitive. À nouveau, l'appareil est conçu par Couffinhal. Pour ne plus être accusé de produire à des coûts plus élevés que les équipements concurrents, il entre dans une logique de recyclage, avec la possibilité d'utiliser en circuit fermé l'eau nécessaire au chauffage de la pâte. Surtout, partant du principe que les houilles présentent toutes des caractéristiques différentes, de dureté notamment, il est préférable de donner davantage de souplesse au matériel, de façon à ce que la pression exercée puisse varier en fonction du charbon utilisé16. Mais se doter d'équipements brevetés représente un coût non négligeable, notamment pour une houillère dont la rentabilité est faible ${ }^{17}$. 
Figure 2. Plan du carreau de la mine de La Chazotte, dans la Loire. Fonds École nationale supérieure des mines de Paris

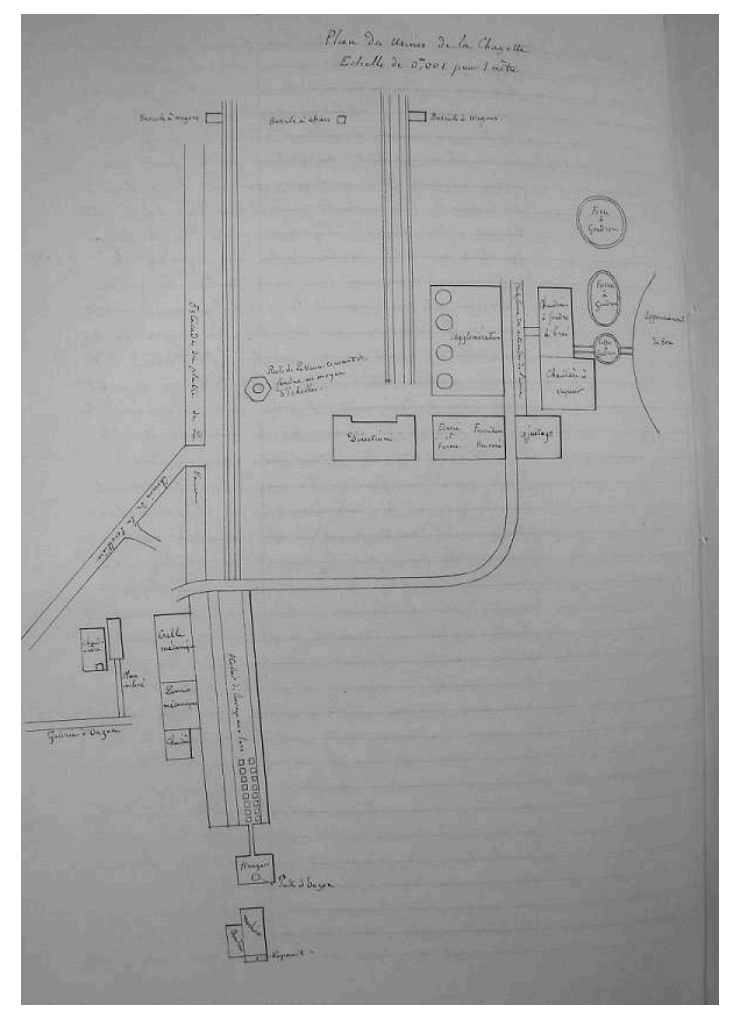

Le plan comprend les installations minières de surface, mais aussi les ateliers annexes et, en particulier, ceux de valorisation de la houille, avec une place très importante dévolue au lavage du charbon. Le site est caractérisé aussi par la présence d'installations de récupération des sousproduits, activité dans laquelle le bassin stéphanois fait figure, en France, de pionnier.

(c) Jean-Philippe Passaqui

Le directeur des mines de La Chazotte, Maximilien Evrard, conçoit donc les appareils qu'il emploie pour valoriser ses charbons. Les quelques années qui le séparent de l'invention de Bérard correspondent à une élévation du prix de la main-d'œuvre, mais aussi des goudrons et des brais. Pour y faire face, il cherche d'abord à améliorer l'efficacité productive des ouvriers de tous les équipements qu'il est amené à installer. En amont de ses propres dispositifs d'agglomération, il modifie les ateliers de lavage existants. Comme certains de ses contemporains, il agit à la fois comme ingénieurdirecteur d'une exploitation, mais aussi comme inventeur, capable d'assurer la promotion de ses installations, après les avoir mises au point et brevetées. Il propose une étude très documentée de son procédé de lavage dans le Bulletin de la société de l'industrie minérale et vend son procédé à travers l'Europe où il est employé jusqu'en Autriche. Ses inventions sont d'ailleurs omniprésentes dans cette publication. Au sein du tome IV, Évrard présente les deux installations de fabrication d'agglomérés dont il est à l'origine ${ }^{18}$.

Pourquoi les mines de la Chazotte sont-elles devenues, au-delà de la personnalité et des talents d'Evrard, le lieu de mise au point et de validation pratique de beaucoup des équipements de valorisation de la houille, depuis sa purification jusqu'à son agglomération? C'est la conséquence d'une situation paradoxale. Les mines de la Chazotte présentent la particularité, dans un bassin stéphanois plutôt réputé pour la qualité de ses houilles, de proposer des charbons médiocres. Friables et barrés par la 
présence de schistes intercalés, leur combustion est mauvaise et accompagnée de cendres et de fumées. Sans valorisation, ce charbon ne peut pas se confronter à celui des autres exploitations stéphanoises. Il est donc celui qui, en proportion, est le plus soumis à transformation.

Cela explique pourquoi, des ateliers de lavage à ceux d'agglomération, La Chazotte se présente comme une exploitation à part, pionnière, qui a su gagner la fidélité d'une certaine clientèle, celle des compagnies de chemins de fer en particulier. C'est d'ailleurs au moment où elles sortent de la crise qui a interrompu leur croissance en 1847 qu'Évrard s'engage dans ses recherches. Il dirige l'exploitation de 1852 à 1872 et construit un premier atelier d'agglomération en 1855, dont la qualité des produits est reconnue lors de l'Exposition universelle de Londres, en $1862^{19}$. Lieu de perpétuelle réflexion technique, La Chazotte aiguise la curiosité des contemporains d'Évrard. Il en fait un site où accourent les élèves des écoles des mines de Paris comme de SaintÉtienne, toujours à la recherche du changement technique. Ils y trouvent un autre intérêt, celui de pouvoir comparer les dispositifs décrits dans les grandes revues techniques avec ceux effectivement construits car ils découvrent qu'en l'espace de quelques années, les modifications peuvent être spectaculaires ${ }^{20}$.

Retrouvons Évrard. Remarquable concepteur, il est en outre le premier utilisateur de ses machines. Sa formation d'ingénieur lui permet d'associer, dans une même démarche, les dimensions techniques et économiques. Ils accordent une place essentielle au calcul dans la mise au point de leurs équipements. Ils expérimentent, comparent et améliorent sans cesse les dispositifs qu'ils imaginent ${ }^{21}$. Ce ne sont jamais des machines pensées et assemblées une fois pour toutes. Or, l'enjeu est de taille. Les appareils d'agglomération sont d'une mise au point complexe. Ils ne sont d'ailleurs en rien révolutionnaires dans leur conception et empruntent beaucoup à différents équipements déjà sur le marché. Évrard comme Révollier s'inspirent de dispositifs imaginés en Angleterre, notamment pour la circulation mécanique des substances, ainsi que pour leur chauffage.

Leurs machines sont en mesure d'associer plusieurs types de charbon, un liant, goudron ou/et brai ainsi que de la vapeur, tout en donnant à l'ensemble un mouvement à la régularité métronomique. Ce mélange est un gage de régularité et d'homogénéité. À ces personnages déjà évoqués et sortis du sérail stéphanois pourrait être associé, dans le domaine de l'agglomération de la houille, Baroulier. Ce dernier connaît au parcours un peu différent. Pendant qu'il s'ingéniait à mettre au point et à industrialiser son propre procédé d'agglomération ${ }^{22}$, il restait, en parallèle, répétiteur à l'École des mines de Saint-Étienne ${ }^{23}$. L'échec de son procédé ne provient pas de la qualité de la briquette obtenue, mais d'un sens pratique qui fait défaut. Le fonctionnement de sa machine s'accompagne d'un personnel pléthorique, aspect qui s'oppose aux buts industriels des équipements déjà en usage. Évrard en faisait un élément cardinal de la conception et de la mise au point de son propre dispositif. Comme ce dernier, il avait imaginé un procédé de compression qui serait suffisamment efficace pour se passer de matière collante $^{24}$.

La briquette obtenue ne laissait en rien à désirer, mais sa production resta confidentielle, ce qui ne permit pas d'en abaisser le coût en jouant sur la quantité. Ses agglomérés restaient donc plus onéreux que ceux obtenus avec les équipements concurrents, ce qui était cocasse puisque l'absence de brai dans le dispositif de compression devait, au contraire, donner au procédé Baroulier un avantage majeur... 
qu'il n'obtint jamais. Cela n'empêche pas la mise au point et l'utilisation des équipements d'agglomération de donner aussi une autre image de l'industrie stéphanoise, souvent cantonnée à l'extraction de la houille et à l'élaboration de la fonte et de l'acier. En fait, les machines d'agglomération participent, comme celles d'extraction, à une véritable réflexion quant aux économies d'échelles, de personnel et de combustibles.

Figure 3. Coupe de l'atelier d'agglomération de La Chazotte. Fonds École nationale supérieure des mines de Paris.

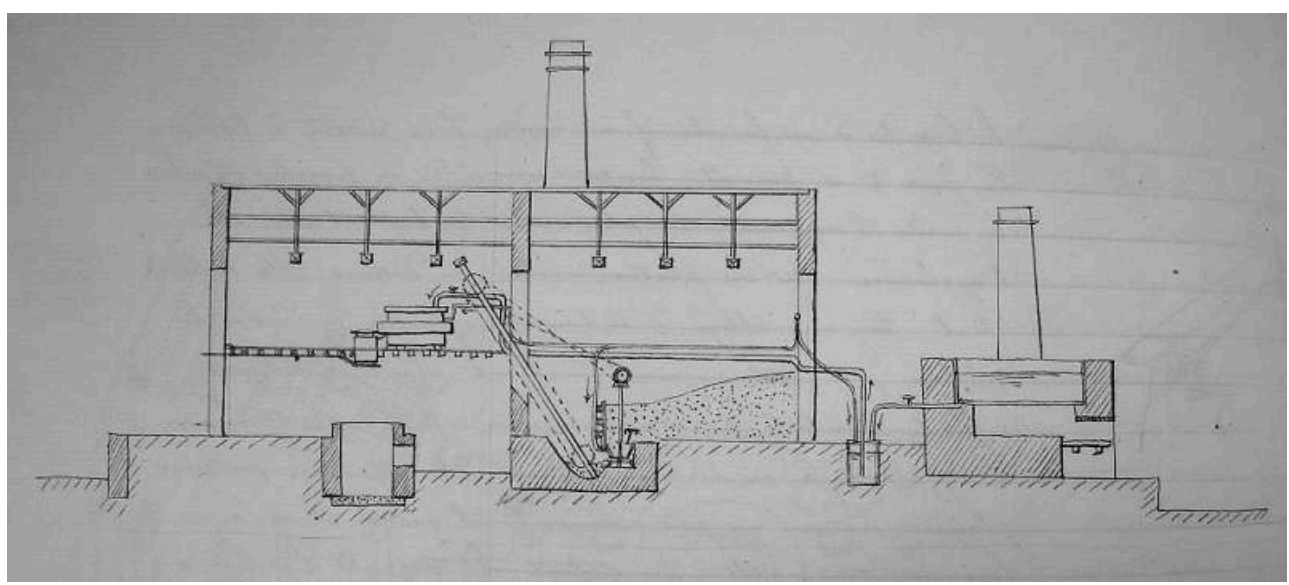

La partie droite du dessin fait ressortir une chaudière dans laquelle le brai ou le goudron sont fluidifiés par la chaleur, avant de pouvoir s'agglutiner aux charbons broyés et former la pâte qui sera ensuite portée vers les presses à agglomérer..

(C) Jean-Philippe Passaqui

21 Face à des contraintes multiples, les industriels du bassin ont fait preuve d'un esprit innovant exacerbé. Autour de la houille, l'environnement technique qui émerge à Saint-Étienne est remarquable. Il se signale par plusieurs inventions décisives dans le domaine du lavage et du triage de la houille, de l'agglomération des charbons, qui ont ensuite essaimé dans toute l'Europe continentale. Faute de pouvoir disposer de ressources suffisantes, la France reste tributaire de ses importations. Ses industriels souffrent d'avoir à utiliser un combustible plus cher qu'ailleurs. Mais, en retour, l'industrie française apprend à utiliser au mieux les ressources dont elle dispose, en valorisant la houille et en attachant une grande attention à la réduction de la consommation par les machines à vapeur. Pour le bassin stéphanois, à un moment où son poids dans l'industrie houillère française décline, et alors que ses réserves sont comptées, proposer son charbon sous forme d'agglomérés lui permet, en quelque sorte, d'ennoblir cette matière, avec un produit de qualité, fruit d'une réflexion technique poussée.

L'industrialisation de l'agglomération de la houille ne doit donc pas être étudiée seule, mais plutôt associée à tout l'environnement énergétique qui se structure autour de l'usage des combustibles minéraux ${ }^{25}$. Car la fabrication de briquettes a un effet cascade en entraînant l'amélioration voire la mise au point d'autres équipements, comme les concasseurs, les broyeurs, afin que les grains de charbon présentent la plus grande homogénéité possible, gage de cohésion. Elle aboutit à une réflexion sur la conception même de ces équipements. Chaque bassin charbonnier possède ses propres constructions et les mines disposent souvent de leurs ateliers. Mais dans le cas de l'agglomération de la houille, les fabricants vers lesquels se tournent les 
agglomérateurs potentiels sont en fait au nombre de trois, deux à Saint-Étienne et un au Havre. Il faut proposer aux clients des machines dont les principaux organes sont robustes et donc d'un entretien simple à entretenir. En cas de rupture, ils doivent être faciles à remplacer, sans interrompre inutilement la production. Car la mécanisation présente ce revers d'être particulièrement dépendante de la solidité des équipements industriels. Une des compagnies implantées dans le bassin de Graissessac en fait les frais, étant obligée d'interrompre pendant plusieurs semaines la production de coke, faute d'avoir su entretenir son lavoir mécanique ${ }^{26}$. C'est d'ailleurs un des principaux aspects du processus de sélection, ou tout au moins une des pistes d'amélioration des différents équipements.

\section{Des installations de surface profondément modifiées par les étapes de la valorisation de la houille}

Comment réagissent les houillères et les grands consommateurs de houille qui font le choix d'utiliser des agglomérés plutôt que de la houille crue? Si les différents équipements peuvent entrer en concurrence, ils sont souvent utilisés simultanément par les grands exploitants charbonniers, dans la mesure où les résultats obtenus permettent de proposer des produits complémentaires. En fait, les compagnies minières se dotent rarement, quand leur production est importante, d'un seul type de machines. Elles se mettent en capacité de répondre à une clientèle qui, pour la navigation ou le transport par rail, manifeste des exigences spécifiques. Quel que soit le procédé, les compagnies houillères peuvent proposer aux compagnies de navigation et ferroviaires un combustible artificiel compétitif par rapport au "charbon en roche" britannique. Il suffit pour s'en convaincre de suivre les pratiques de la Marine impériale. Par habitude, jusqu'à l'avènement de l'agglomération, elle utilise des combustibles britanniques et regarde avec une certaine défiance les houilles françaises ${ }^{27}$. En associant une meilleure préparation de la houille, par une classification rigoureuse de celle-ci, à la production d'agglomérés de qualité, les industriels français sont en mesure de gagner un marché, celui de l'approvisionnement de la flotte de Toulon, qui leur échappait complètement au profit des charbons britanniques ${ }^{28}$. Ils réservent pour les ports leurs meilleurs charbons retirés du triage pendant que, de plus en plus, ils apprennent à respecter les cahiers des charges imposés par la flotte française.

Pour parvenir à bonnes fins, outre la confrontation minutieuse des différentes solutions techniques, les compagnies houillères se dotent de laboratoires dans lesquels elles peuvent réaliser les expériences afin d'améliorer leurs produits. Les compagnies minières développent aussi le contrôle a priori et a posteriori de leurs productions, en s'équipant d'installations capables d'en garantir la qualité au moment du chargement, afin de ne pas se retrouver en contradiction avec les résultats du client, notamment pour les briquettes ${ }^{29}$. Elles sont capables de maintenir et d'améliorer; maintenir en créant les conditions d'une production régulière, celle pour laquelle un contrat a été signé avec un client et améliorer, en étant à même, tout en conservant la qualité initiale du produit, de chercher et soumettre des améliorations. L'idée étant que la qualité des agglomérés, au pire, soit stable, mais puisse aussi être bonifiée.

Les mines de Portes, dans le Gard, mettent au point un appareil pour mesurer rapidement le degré de cohésion de la houille, ce qui leur permet d'obtenir de meilleurs 
résultats que celles de la Grand-Combe. Exploitations secondaires, elles remportent grâce à cette initiative des marchés avec la Marine, plus exigeante que les compagnies de chemins de fer. Au moment où les houillères se dotent d'ateliers d'entretien et, ce faisant, doivent recruter un ingénieur mécanicien, un chimiste devient aussi indispensable pour mesurer chaque jour la composition des houilles, du coke et des briquettes ${ }^{30}$.

Un autre aspect aussi décisif apparaît ${ }^{31}$. Pour les houillères, adopter des équipements de lavage, de carbonisation et d'agglomération de la houille présentant un fort degré de mécanisation engendre des effets au fond. L'essentiel de la main-d'oeuvre masculine y est concentré, la surface regroupant un personnel de jeunes et de vieux ouvriers, dont la productivité du travail est faible. Cantonné à des tâches répétitives qui ne nécessitent pas une grande force physique, ils se retrouvent en concurrence avec la main-d'oeuvre féminine. La mécanisation des installations du jour ne se fait pas au détriment de l'emploi. Elle coïncide plutôt avec des phases de croissance du personnel ouvrier. Ainsi, à La Chazotte, la généralisation du lavage mécanique et de l'agglomération de la houille correspond à une multiplication par dix du nombre d'ouvriers, essentiellement dans les travaux miniers ${ }^{32}$. Car, dans les ateliers d'agglomération, le personnel est pratiquement absent, la circulation des matières se faisant exclusivement par le mouvement des pistons, des vis et des godets. Ce qui étonne, c'est la minutie, la précision avec laquelle vapeur, brai, goudron et charbon s'écoulent pour produire des briquettes.

Il se forme une opposition flagrante entre les deux mondes de la mine. Le fond, hormis pour l'élévation du charbon, reste un lieu peu mécanisé, où le pic demeure l'outil de base, même lorsque, au cours des années 1860-1870, les premiers équipements d'abattage mécanique entrent en activité. Au jour, au contraire, le personnel est fortement réduit, pour éviter que les frais d'extraction, difficilement compressibles, ne soient amplifiés par le fonctionnement des équipements de surface. La main-d'œuvre y perd, pour la majeure partie d'entre elles, toute qualification. Elle est spécialisée dans des tâches répétitives. Sa rémunération dépend de l'efficacité du fond. Elle est de toute façon modique. Ce faisant et sur certains points, le fonctionnement des ateliers de surface préfigure l'organisation productive fordiste. Le triage fait appel à des convoyeurs à bandes le long desquels sont réparties des rangées de femmes, les trieuses, pour éliminer les roches, schistes et autres matières stériles qui accompagnent la houille. Il s'agit d'un travail cadencé, immobile. La matière circule devant les ouvrières qui, en quelque sorte, la désassemblent. Pour l'agglomération, le raisonnement est inversé. Le charbon, après avoir été broyé, est mélangé avec le brai, sans aucune intervention de personnel. Le travail est complètement mécanisé. Quant à l'agglomération proprement dite, elle nécessite un contrôle de la qualité des produits, de la régularité du fonctionnement de la presse. Il s'agit d'assembler et de donner de la consistance à une pâte composée de houille et de brai. La substance manipulée est, dans sa composition, simple, mais la logique de la production s'apparente déjà à une véritable chaîne ${ }^{33}$. En outre, l'aménagement des équipements, en réduisant au maximum l'intervention humaine, en tire un surcroît de qualité. Les équipements sont agencés de façon à infliger le moins de chocs possible au charbon et aux briquettes, avec des mouvements doux, réguliers. 
Figure 4. Coupe de l'atelier d'agglomération de La Chazotte, machine à agglomérer. Fonds École nationale supérieure des mines de Paris.

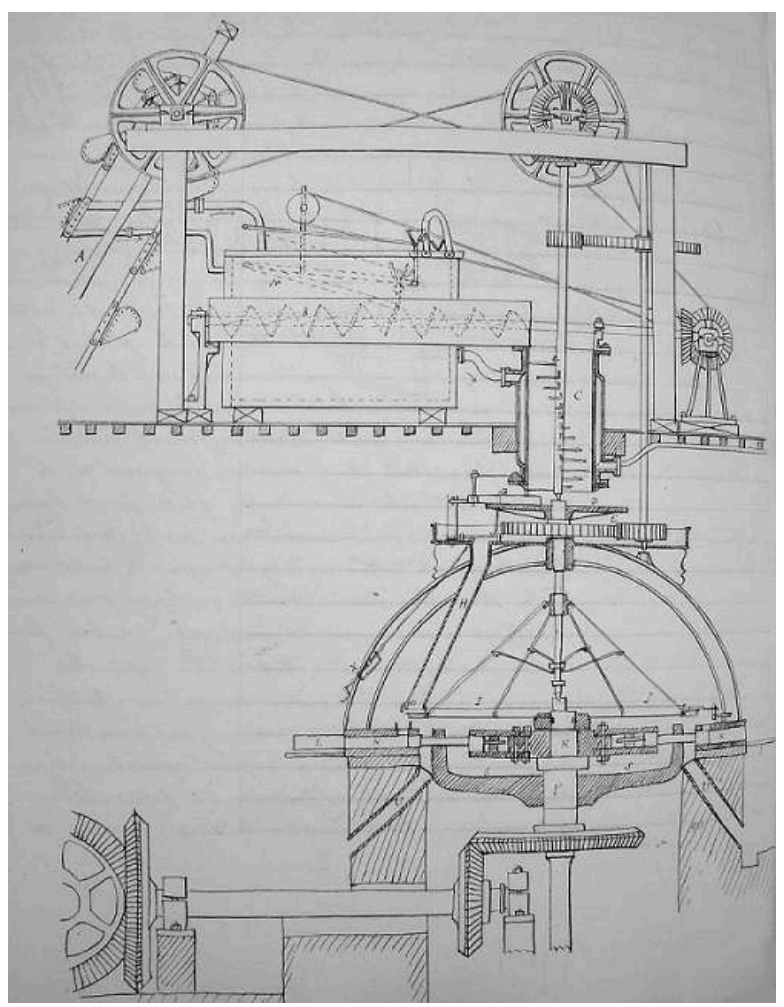

Cette partie de l'atelier se signale par un machinisme très développé, et par un cadencement très strict des matières, à la fois en termes de vitesse de circulation et de mélanges entre la houille et le brai. La marche est continue. Une machine à vapeur communique un mouvement de va et vient aux pistons qui apportent et retirent le mélange des presses dans lesquelles s'opère l'agglomération.

(c) Jean-Philippe Passaqui

Ce sont donc deux mondes qui s'opposent, celui qui crée l'essentiel de la richesse, avec l'abattage et l'extraction de la houille et celui qui s'est ajouté au jour. Le modèle technique sur lequel il repose en garantit la réussite économique, grâce à un effort de mécanisation de tous les instants. Pour reprendre l'expression de l'élève externe Savignac : «tout se fait par la machine à vapeur ${ }^{34}$ » dans les ateliers de fabrication de briquettes. Ainsi, à la Grand-Combe, la compagnie aborde le début des années 1870 avec des ateliers de lavage mécanisés, des fours à coke à défournement mécanique ${ }^{35}$. Au sein de ces ateliers, les conditions de production d'agglomérés deviennent essentielles.

\section{Conclusion}

Disposer d'un produit connu, à la composition homogène, constitue un atout essentiel en vue de s'attacher la clientèle industrielle. La mise au point et le retour d'expériences sont fondamentaux pour comprendre le succès presque immédiat de la filière. Dans ce dessein, les compagnies de chemins de fer produisent une partie leurs agglomérés. Elles conservent en parallèle des relations contractuelles durables avec des fournisseurs soigneusement identifiés et contrôlés. Elles peuvent donc les épauler dans l'élaboration des agglomérés. C'est ce qui explique pourquoi, partant de blocs de grosses dimensions, les agglomérés perdent ensuite en épaisseur et en taille. Il est possible de faire mieux qu'imiter les meilleures houilles. La marine et les chemins de fer demandaient des 
fragments homogènes dans leur composition, mais aussi dans leur taille. À ce titre, le fait que la compression permette de gagner un tiers du volume n'est pas anodin dans la réussite de ce procédé. Des solides réguliers, cylindriques ou parallélépipédiques, peuvent être empilés avec facilité dans les soutes à charbon ${ }^{36}$. Ils ne modifient pas les caractéristiques du charbon si les principaux critères suivis avec attention par le consommateur, c'est-à-dire l'indice de gonflement, le ramollissement, l'indice en matières volatiles et le comportement au feu respectent les cahiers des charges correspondant aux besoins du client. Les techniques développées dans le cadre de la valorisation de la houille ne restent pas confinées. Elles sont reprises par d'autres activités. Au cours des années 1860, l'agglomération inspire l'industrie de la carbonisation du bois, ainsi que l'agglomération de certains minerais de fer et des pyrites.

\section{NOTES}

3. CHALMETON Ferdinand, "Note sur l'industrie houillère du département du Gard », Bulletin de la Société de l'industrie minérale, $1^{\text {re }}$ série, t.XII, 1866-1867, p.120.

4. Le PLM est en mesure de produire ses propres agglomérés à partir d'usines dont il dispose dans le Centre et le Midi de la France. Il a notamment racheté le site de La Chazotte, en 1874, pour sécuriser ses approvisionnements.

5. GAUTIER Ferdinand, Mémoire sur le bassin houiller de Brassac (Puy-de-Dôme, Haute-Loire), ENMSP, ms, 1862, M (1862) 780.

6. PASSAQUI Jean-Philippe, «Les compagnies minières du Centre et du Midi de la France face aux difficultés engendrées par les couches puissantes de houille (1850-1873) », Actes du colloque des machines et des hommes, Lewarde, 2013, p.44-51 et 179-182.

7. SOUGY Nadège, «Le combustible minéral au XIX ${ }^{\mathrm{e}}$ siècle, une affaire de qualité », Nomenclatures et classifications : approches historiques, enjeux économiques, Paris, INRA, 2003, p.69-96.

8. D'où l'importance que Louis Grüner accorde à cette question au moment de fonder la Société de l'industrie minérale et le bulletin qui l'accompagne. Voir GARÇON Anne-Françoise, Entre l'État et l'usine, l'École des mines de Saint-Étienne au XIX siècle, Rennes, Presses Universitaires de Rennes, 2004, p. 252. Il fallut cependant attendre 1864 pour que Grüner publie un article de référence sur la question de l'agglomération de la houille.

9. GRÜNER Louis, « Notice sur l'agglomération des combustibles minéraux », Annales des Mines, 1864, p.150.

10. GRÜNER Louis, « Notice... », op. cit., 1864, p.188-190.

11. Charles Combes, alors inspecteur général des Mines, est le premier à conseiller aux exploitants des Cévennes de valoriser leurs menus de houille sous la forme d'agglomérés. Archives nationales (AN) F14/3835, Rapport de l'ingénieur des Mines du sous-arrondissement minéralogique d'Alais sur la situation des mines du département du Gard en 1852.

12. Émile Marsais est de la promotion 1828 de l'EMSE. Ses premiers travaux interviennent en 1833, mais ce n'est qu'en 1842-1843 qu'il parvient, avec des équipements fabriqués par Révollier, à donner une dimension pratique à la production d'agglomérés. Il est à l'origine, en 1846, de l'usine de Givors évoquée. LESEURE Ernest-Louis, Historique des mines de houilles du département de la Loire, Saint-Étienne, Théolier, 1901, p.296-297. 
13. Extrait cité par GRAS Louis-Joseph, Histoire économique générale des mines de la Loire, t. I, SaintÉtienne, Théolier, 1922, p.367-368, mais la mise en avant de l'agglomération est encore timide comme le prouve la place anecdotique qu'elle occupe dans les rapports du Jury de l'Exposition universelle de Paris, en 1855.

14. Ibidem, p. 303.

15. L'entreprise s'appelle alors Révollier, Biétrix et Cie. Elle est implantée à La Chaléassière. « État des ingénieurs », Bulletin de la Société de l'industrie minérale, 1875.

16. GARNIER Jules, " Nouvelle machine à agglomérer de MM. Biétrix et Cie », Bulletin de la Société de l'industrie minérale, 1877-1878, p.190.

17. Comme nous le verrons, le charbon de La Chazotte est à la fois cendreux et pulvérulent, dans un bassin où la concurrence est en mesure de proposer un charbon de bien meilleure qualité.

18. ÉVRARD Maximilien, « Notice sur l'usine d'agglomération de La Chazotte (Loire) », Bulletin de la Société de l'industrie minérale, $1^{\text {re }}$ série, t. IV, 1859, p.261.

19. GRAS Louis-Joseph, Histoire économique générale des mines de la Loire, t. II, Saint-Étienne, Théolier, 1922, p.743-744.

20. À ce sujet, GARÇON Anne-Françoise, "Fours debout, fours couchés ", L'imaginaire et la pensée technique, Paris, Classiques Garnier, 2012, p.131-148.

21. VOISIN Armand, Mémoire sur l'exploitation, le lavage et l'agglomération de la houille à la mine de La Chazotte, près Saint-Étienne, ENMSP, ms, 1863, M (1863) 810.

22. Brevet déposé le 24 août 1855, production en 1857 et 1858 .

23. L'article que Grüner publie en 1864 dans les Annales des Mines, à propos de l'agglomération de la houille, évoque longuement les travaux de Baroulier, en restant assez pudique sur les causes de leurs échecs industriels. La mise en exergue des recherches de Baroulier dans ce domaine doit beaucoup à nos discussions avec Marco Bertilorenzi ; qu'il en soit remercié. Outre ses expériences industrielles à Saint-Étienne, Baroulier a aussi dirigé la création et le développement de l'agglomération de la houille à Blanzy.

24. VOISIN Armand, Mémoire..., op.cit., 1863.

25. ROJAS Luc, Histoire de révolution technologique, de l'exploitation artisanale à la grande industrie houillère de la Loire, Paris, L'Harmattan, 2008, p.155-158.

26. NORMAND Charles, Journal de voyage dans le sud de la France, ENSMP, ms, 1860, JV (1860) 229. Il s'agissait d'une machine Bérard.

27. PASSAQUI Jean-Philippe, « Aux origines des perturbations des marchés charbonniers par les conflits et les expéditions militaires : la déstabilisation des houillères du Midi de la France par la guerre de Crimée ", Charbon et conflits dans le monde, Actes du colloque international de Lewarde, 2016, p.88-99.

28. CHALMETON Ferdinand, « Note... », op.cit., 1866-1867, p.120-123.

29. CHODZKO Adam, Journal de voyage, ENSMP, ms, 1872, JV (1872), 459.

30. BENOIST P., Journal de voyage dans le Centre et le Midi de la France, houillères de Blanzy, juillet, août, septembre 1873, ENSMP, ms, 1873, JV (1873) 488.

31. RIGAUD Fernand, Exploitation de la houille dans le bassin de Saint-Étienne, ENMSP, ms, 1868, M (1868) 874 .

32. VOISIN Armand, Mémoire sur l'exploitation, le lavage et l'agglomération de la houille à la mine de La Chazotte, près de Saint-Étienne, ENSMP, ms, 1863, M (1863) 810.

33. WORONOFF Denis, "Charbon et sidérurgie: l'installation en France du nouveau système productif», Actes du colloque Travailler à la mine, une veine inépuisée, Arras, Artois Presses Université, 2003, p. 113.

34. SAVIGNAC Louis de, Journal de voyage, exploitation de Campagnac, ENSMP, ms, 1872, JV (1872) 465.

35. PASSEBOIS Marius, Journal de voyage, $2^{e}$ partie, 1872, ENSMP, ms, 1872, JV (1872) 465. 


\section{RÉSUMÉS}

Valoriser les déchets, les commercialiser a toujours été une préoccupation d'importance pour les entreprises minières, et notamment les houillères. Au milieu du XIX ${ }^{\mathrm{e}}$ siècle, dans l'ensemble de l'Europe occidentale, mais plus particulièrement en France, une tendance lourde affecte les différentes exploitations qui tentent de mettre au point et d'utiliser des procédés qui permettent l'agglomération des charbons de petits calibres, afin de pouvoir les écouler auprès des compagnies de chemins de fer et de navigation. Le bassin stéphanois et avec lui les exploitations du Centre et du Midi de la France s'engagent avec ardeur dans cette voie pour en faire une spécialité rémunératrice. En définitive, une nouvelle coordination de l'activité productive doit s'imposer, qui repose sur une mécanisation précoce, intense, faisant apparaître un contraste évident avec les travaux souterrains où le pic domine toujours.

Recovering and marketing waste has always been a major concern for mining companies, especially collieries. Giving economic value to small coals in the way to send them to railways and shipping companies became a major trend in mining companies, in the middle of the 19th century Europe, particularly in France. The Saint-Étienne collieries has been eagerly engaged in this way to make it a profitable activity. Eventually, a new co-ordination of the productive activity imposed itself in the mining companies of the center and the south of France, which was based on an early and intense mechanization, strikingly contrasting with the manual mining, still dominating in underground works.

\section{INDEX}

Mots-clés : histoire des techniques, histoire des entreprises, charbon, valorisation des déchets, district industriel, XIXe siècle, France

Index chronologique : Époque contemporaine Index géographique : France

Thèmes : Un objet une technique

Keywords : history of technology, business history, collieries, waste recovery, XIXth century, France

\section{AUTEUR}

\section{JEAN-PHILIPPE PASSAQUI}

Jean-Philippe PASSAQUI est chercheur affilié au Centre d'histoire des techniques, IHMC UMR 8066, Université Paris 1 Panthéon-Sorbonne/ENS Ulm/CNRS. Agrégé d'histoire et docteur en histoire contemporaine, il a obtenu le prix spécial d'histoire de l'Académie François-Bourdon en 2003. Il est par ailleurs professeur d'ESHMC en CPGE voie ECE, option Saint-Cyr au Lycée Militaire d'Autun. Spécialiste d'histoire des entreprises et de l'industrie, il a notamment publié La Stratégie 
des Schneider, du marché à la firme intégrée en 2006, (réed. 2015) et Les Voyages forment l'ingénieur, les houillères du Centre et du Midi de la France, 1851-1873, Paris, Classiques Garnier, en 2015. Avec Marco Bertilorenzi et Anne-Françoise Garçon, il a dirigé les actes du colloque Entre technique et gestion, une histoire des ingénieurs civils des mines, $\mathrm{XIX}^{e}-\mathrm{XX}^{e}$ siècles, Paris, Presses des Mines, 2016 et achève ceux du colloque Henri Fayol, histoire, économie et sciences. Sous le parrainage d'Anne-Françoise Garçon, il prépare actuellement son HDR. 